\title{
High throughput RNAi to study the interaction between barley and powdery mildew fungi
}

\author{
D. Douchkov, W. Dong, U. Zierold, D. Nowara, U. Seiffert and P. Schweizer \\ Institute of Plant Genetics and Crop Plant Research (IPK), D-06466 Gatersleben, Germany. \\ Correspondence to: P. Schweizer, Institute of Plant Genetics and Crop Plant Research (IPK), D-06466 Gatersleben, Germany, \\ E-mail: schweiz@ipk-gatersleben.de \\ Received: September 5, 2005
}

Barley is one of the most important feed and food crops worldwide. Despite its agronomic importance and excellent, available genetic resources, tools for genome-wide analysis of barley have only recently been initiated and include high-resolution genetic maps, physical gene mapping, highly efficient protocols for genetic transformation, insertion mutagenesis, TILLING platforms, a large EST collection as well as gene arrays for expression profiling. We have contributed to extending this genomics toolbox in barley by establishing 22,000 EST sequences from powdery mildew-attacked barley epidermis, a 10K cDNA array as well as a high-throughput RNAi system for assessing gene function in attacked barley epidermal cells. The RNAi system for transient-induced gene silencing (TIGS) based on biolistic transgene delivery is being used to study the function of approximately 900 barley candidate genes including 693 up-regulated genes, 101 resistance-gene analogues expressed in barley epidermis as well as 58 proteasome component genes. The library of RNAi constructs was built up by a new, cost-efficient method that combines highly efficient ligation and recombination by the GATEWAY cloning system into a final RNAi destination vector that was found to direct highly efficient RNAi. The full RNAi construct library was tested in a TIGS screening for breakdown of nonhost resistance against wheat powdery mildew. Approximately 200 up-regulated host genes were also tested for breakdown of mlo-mediated host resistance or modulation of host susceptibility. Forty-three candidate genes producing a susceptible or resistant phenotype in one or several of the first-round TIGS screening are being analyzed in greater detail. Until present, ten genes were found to alter (non)host responses of barley to powdery mildews in a reproducible manner. 